\title{
Assessment of Cardiovascular Surgical Infection Prophylaxis in a Lebanese Hospital
}

\author{
Mary Younes, Diana Malaeb and Mohamad Iskandarani \\ Department of Clinical Pharmacy, Lebanese International University, Beirut 362060, Lebanon
}

\begin{abstract}
The appropriate use of antibiotics prophylaxis reduces the incidence of surgical site infections. Despite advances in antiseptic measures, antibiotics, and preoperative precautions, infections are common complications from surgeries. This study was conducted to assess the appropriateness of the prescription of antibiotics prophylaxis prior to cardiac surgery among hospitalized patients in the geographic area of Lebanon. This was a retrospective, observational, single center study conducted at a public Lebanese teaching hospital from February till April 2014. Data were collected from computerized data bases for hospitalized patients who did cardiac surgeries from January 2010 till December 2013. Patients above eighteen years old undergoing CABG (coronary artery by-pass graft), valve surgery, or both were eligible for study enrollment. Excluded were patients younger than eighteen years of age, prior intake of antibiotics, or had another concomitant surgery with the cardiac. Consistency with the guidelines was evaluated for appropriate route of administration, choice, preoperative timing, duration, dosing, and redosing of antibiotics. The IRB (Institutional Review Board) approved the study design. Data were analyzed by the SPSS version 20.0 and presented as frequency/percentage and mean \pm SD (standard deviation). A total of 3,000 patients were initially screened where only 245 patients met the inclusion criteria. Baseline age of the participants was $58.91 \pm 13.65$ years (mean \pm standard deviation SD), and a body mass index of $28.19 \pm 5.19 \mathrm{Kg} / \mathrm{m}^{2}$. The enrolled participants were on different intravenous antibiotic regimens, where $188(76.7 \%)$ patients were on vancomycin and ceftriaxone, 15 $(6.1 \%)$ on vancomycin and cefuroxime, $13(5.3 \%)$ on ceftriaxone, $12(4.9 \%)$ on vancomycin, $6(2.4 \%)$ on cefuroxime, $2(0.8 \%)$ on clindamycin), and the others were on combination of gentamicin and a cephalosporin. Only twelve (4.9\%) from the enrolled patients were consistent with the treatment guidelines regarding the choice of the antibiotic. For those patients who were given the appropriate antibiotics, proper dose was found in only seven patients (58.7\%). Five patients out of $254(2 \%)$ required redosing where four were given the appropriate doses. As for preoperative timing it was appropriate for all of those cases (i.e. within 120 minutes of incision for vancomycin and within 60 minutes for cefuroxime and clindamycin). Prophylaxis was extended beyond one day in 173 patients (70.61\%) and the average duration was 2.8531 days \pm 2.07514 . This study demonstrates that in cardiac surgery, the optimal choice of antibiotics is seldom administered, duration of prophylaxis is excessively long, and the preoperative dose timing is rarely employed. More education and communication are required to improve these practices to reduce risks of surgical site infection, prevent resistance, and limit costs potentially associated with antibiotic misuse. The role of clinical pharmacist may facilitate this process across all surgical disciplines through interventions that should be implemented to optimize the perioperative antibiotic prophylaxis in procedures.
\end{abstract}

Key words: Cardiac surgery, antibiotic prophylaxis, surgical site infections.

\section{Introduction}

\subsection{Surgical Site Infections}

Infections that occur within 30 days of the surgery are termed SSIs (surgical site infections). Postoperative SSIs according to Nichols (2001) remain a major source of illness in surgical patients. These infections number approximately 500,000 per year,

Corresponding author: Mary Younes, Pharm. D., research fields: surgical site infections. E-mail: mary_younes@live.com. among an estimated 27 million surgical procedures, and account for approximately one quarter of the estimated 2 million nosocomial infections each year [1].

The criteria used to define surgical site infections have been standardized and described three different anatomic levels of infection: superficial incisional surgical site infection, deep incisional surgical site infection and organ/space surgical site infection (Fig. 1) [2]. 


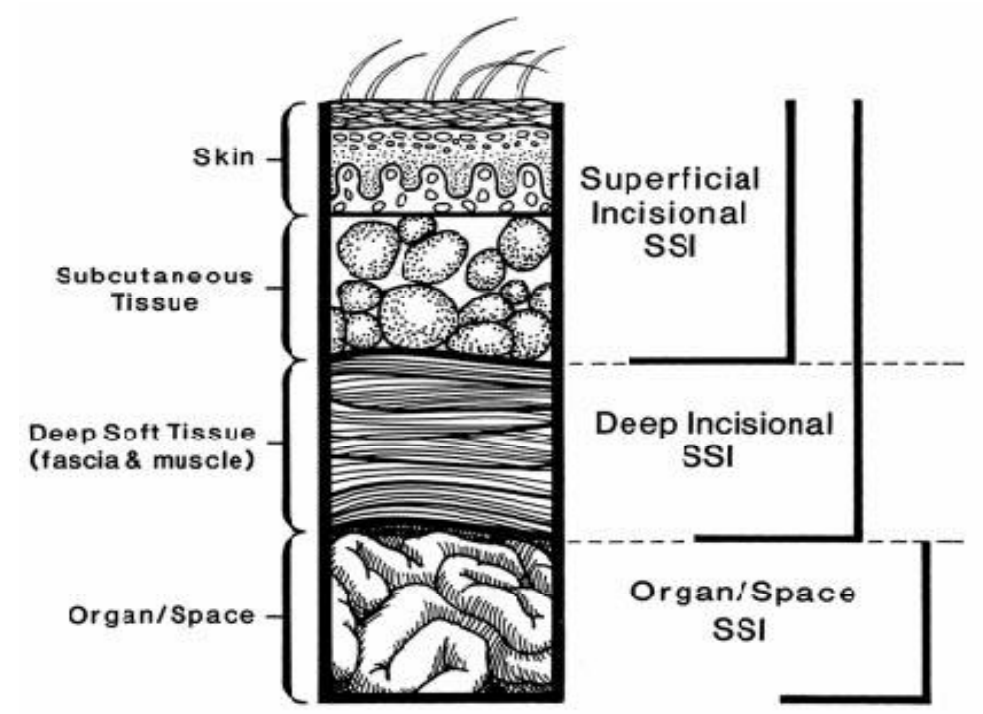

Fig. 1 Cross-section of abdominal wall depicting CDC classifications of surgical site infection [3].

\subsection{Complications after Cardiac Surgery}

Infections, cardiac complications (e.g. arrhythmias and cardiac temponade), neurologic complications (e.g. phrenic nerve injury and paralysis), and pulmonary or renal dysfunction may take place following cardiac surgery.

\subsection{SSIs after Cardiac Surgeries}

\subsubsection{Risk Factors}

SSIs are a serious complication after cardiac surgery with occurrence rate up to 5\%. Risk factors for SSIs after cardiac procedures have been identified from several single-center cohort and case-control studies. These include diabetes, hyperglycemia, PVD (peripheral vascular disease), COPD (chronic obstructive pulmonary disease), obesity (body mass index more than $30 \mathrm{~kg} / \mathrm{m}^{2}$ ), heart failure, advanced age, involvement of internal mammary artery, reoperation, increased number of grafts, long duration of surgery, and Staphylococcus aureus nasal colonization [4-6].

\subsubsection{Infections}

Infections following cardiac surgeries can range from superficial involvement of the wound to deeper soft tissue infection, sternal osteomyelitis, and mediastinitis, which may require surgical drainage and debridement, as well as antimicrobial therapy [4-6].

1.3.3 NNIS (National Nosocomial Infections Surveillance) category of Cardiac Surgeries

In cardiac surgeries the wounds are classified as clean. As for the other NNIS parameters in cardiac surgeries, the $\mathrm{T}$ point is 5 and the American Society of Anesthesiologists score is 3 for most of those procedures. Thus based on some references, NNIS incidence of SSI in cardiac surgery depends mostly on the duration of surgery if it is more than $5 \mathrm{hrs}$. Published NNIS data suggest that the risk of infection by 30 days would be $3.5 \%$ for operations lasting less than $5 \mathrm{hrs}$ and increased to $5.5 \%$ for operations lasting more than $5 \mathrm{hrs}$ [7].

\subsection{Surgical Infection Prophylaxis in Cardiac Surgeries}

\subsubsection{Common Pathogens}

In cardiac surgeries, gram-positives are the most common pathogens found including Staphylococcus aureus, coagulase-negative Staphylococcus, and rarely Propionibacterium acnes [8]. Gram-negative organisms are less commonly isolated in these patients.

\subsubsection{Recommended Regimens}

The recommended regimen in cardiac surgeries for 
SSI prophylaxis, is a single pre-incision dose of cefazolin ( $2 \mathrm{~g}, 3 \mathrm{~g}$ for paients weighing $\geq 120 \mathrm{~kg}$ ) or cefuroxime $(1.5 \mathrm{~g})$ with appropriate intraoperative redosing if the duration of the surgery exceeds two half-lives of the drug or there is excessive blood loss during the procedure, as previously stated. Cefazolin and cefuroxime have a half-life ranging from 1.2-2.2 hrs and 1-2 hrs respectively [9], so redosing is performed if the duration of the surgery exceeds $4 \mathrm{hrs}$ when using those agents.

Clindamycin (900 mg) or Vancomycin $(15 \mathrm{mg} / \mathrm{kg})$ is an acceptable alternative in patients with a documented $\beta$-lactam allergy with a half-life ranging between 2-4 hrs, clindamycin is redosed if duration of the surgery exceeds $6 \mathrm{hrs}$, while vancomycin redosing will not be needed except for unusually long procedures.

The routine use of vancomycin for SIP is not recommended [9], but should be used for prophylaxis in patients known to be colonized with MRSA, or at high risk for MRSA colonization in the absence of surveillance data (e.g., patients with recent hospitalization, nursing-home residents, hemodialysis patients) $[3,9,10]$.

Combine clindamycin or vancomycin with another agent (cefazolin if the patient is not $\beta$-lactam allergic; aztreonam, aminoglycoside, or single dose fluoroquinolone if the patient is $\beta$-lactam allergic), if organizational SSI surveillance shows that gram-negative organisms cause infections for patients undergoing these operations. Mupirocin should be given intranasally to all patients with documented Staphylococcus aureus colonization.

The optimal duration of antimicrobial prophylaxis for cardiac procedures continues to be evaluated. Data support a duration ranging from a single dose up to 24 hours postoperatively.

\subsection{SIP in Lebanon}

There are few studies reporting patterns of SIP in Lebanese hospitals. A previous prospective study in
Lebanon for three months described the adequacy of antimicrobial prophylaxis in 10 acute care hospitals. Conclusion of the study showed that antimicrobial prophylaxis in Lebanese hospitals was fairly adequate in terms of respecting indications for selected surgical procedures, and that improvement could be made by reducing the duration of prophylaxis and avoiding the use of broad-spectrum antibiotics [11].

\subsection{Purpose of the Study}

The appropriate use of prophylactic antibiotics reduces the incidence of surgical site infections. Despite the advances in antiseptic measures, antibiotics, and preoperative precautions, infections are common complications from surgeries. This study was conducted to assess the appropriate prescription of antibiotics prior to cardiac surgery with respect to the choice, dose, duration, and preoperative timing among hospitalized patients in the geographic area of Lebanon.

\section{Methods}

\subsection{Study Design}

This was a retrospective, observational, single center study conducted at a public Lebanese teaching hospital from February till April 2014. Data were collected from computerized medical records for hospitalized patients who did cardiac surgeries from January 2010 till December 2013.

The study protocol and amendments were approved by the institutional review boards of LIU (Lebanese International University) and BGUH (Beirut Governmental University Hospital) where the study was conducted. The analysis of the data was performed basically by the pharmacist.

\subsection{Study Population}

The study took place at the Surgery Unit of BGUH where a total of 3,000 patients who went various types of surgeries were screened and a total of 283 cardiac surgeries included. From the initially enrolled 
participants, 38 patients were excluded for the following reasons: One patient was younger than eighteen years of age, two had other concomitant surgeries with the cardiac, and the rest were taking antibiotics prior to the surgery. Consequently, data were collected and completed for 245 patients.

Patients above eighteen years old undergoing CABG, valve surgery, or both were eligible for study enrollment.

Patients younger than eighteen years of age, previous intake of antibiotics prior to the surgery, or had another concomitant surgery with the cardiac were excluded.

\subsection{Study Procedure}

The data collection started in the hospital after the medical team was informed about the study. The information was taken basically from computerized data bases and the pharmacy department as well. Data collection sheet was created by the clinical pharmacist and the information included was based on the variables that are important to determine the protocols used for surgical prophylaxis. Input included the demographic characteristics of the patients, type and duration of the cardiac surgery, and the surgical infection prophylaxis regimen. Antibiotic regimens were assessed for the following variables: appropriate route of administration, choice, preoperative timing, duration, dosing, and redosing whenever applicable
(Appendix I ).

\subsection{Study Outcomes}

Primary: Evaluate the appropriateness of prophylaxis in comparison with guidelines.

\subsection{Statistical Analysis}

Data were analyzed using the SPSS (statistical package of the social sciences), version 20.0. Discrete variables such as the gender, type of antibiotics, or type of surgery were presented as percentages, however continuous variable such as age or duration of prophylaxis were displayed as mean \pm SD (standard deviation).

\section{Results}

\subsection{Patients' Characteristics}

Data collection was conducted from February to April 2014. A total of 3,000 surgeries between January 2010 and December 2013 were screened, out of which 283 cardiac surgeries were initially included. Only 245 patients were enrolled in the study after meeting the inclusion criteria. From the enrolled subjects, 38 patients were excluded for the following reasons: One of the participants was younger than 18 years old and underwent septal defect closure surgery, two had a urosurgery concomitantly performed with the cardiac, and the rest were on previous antibiotics intake (Fig. 2).

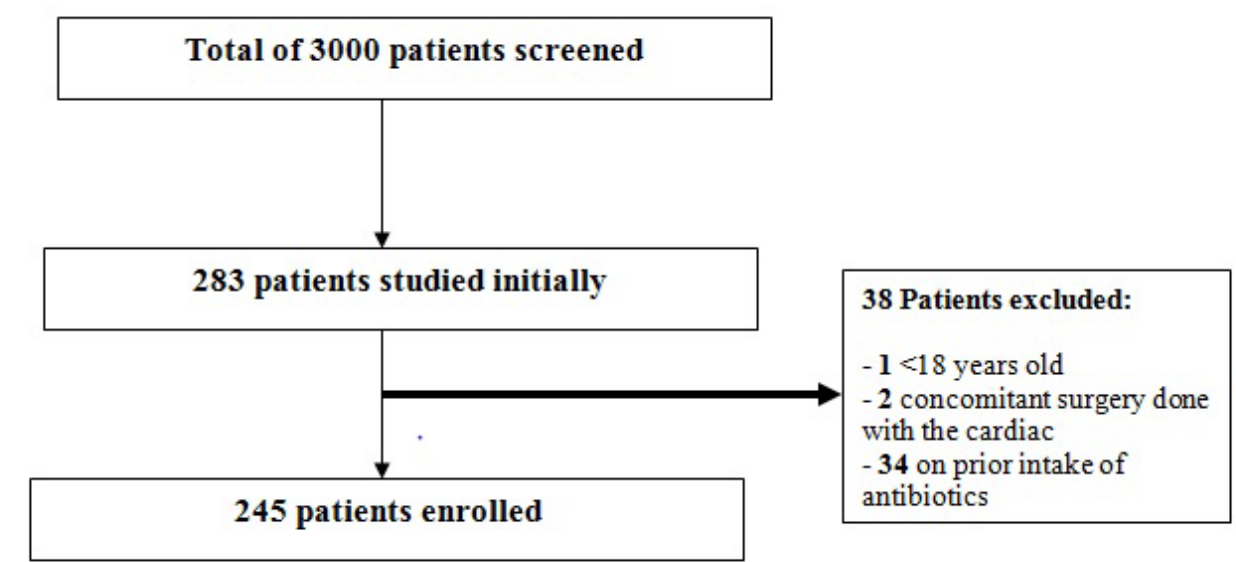

Fig. 2 Patients' flow chart. 


\subsubsection{Gender}

The results show that males were present in a higher frequency than females in the research sample population $(n=245)$. From the total number of patients enrolled, $152(62 \%)$ of the participants were males and $93(38 \%)$ were females. This outcome is consistent with the fact that male gender carries a higher risk for cardiac diseases.

\subsubsection{Age}

Enrolled patients had a mean age of $59.51 \pm 12.15$ (Mean years \pm Standard deviation SD) as displayed in Table 1.

\subsubsection{BMI (body mass index)}

None of the patients was underweight. Patients were divided into normal weight 47 (19.2\%), overweight 84 (34.4\%) or obese $72(29.4 \%)$. The average BMI was $28.19 \pm 5.19$.

\subsubsection{Allergies}

Only $17(6.9 \%)$ of the enrolled patients suffered from an allergy that was either to: PNC (penicillin), contrast dye, ASA (aspirin), sulfa group, or CBZ (carbamazepine). The distribution of the frequencies is presented in Table 2.

\subsubsection{Medical Conditions}

Patients who underwent cardiac surgeries where suffering either from CAD (coronary artery disease) 177 (72.24\%), valvular heart disease 41 (16.73\%), or both 27 (11.02\%). Other common conditions associated with the heart disease were: HTN (hypertension) 159 (64.9\%), DM II (diabetes mellitus type 2) 89 (36.3\%), and DL (dyslipidemia) 55 (22.4\%). The results are summarized in Table 2.

\subsection{Cardiac Surgery Characteristics}

\subsubsection{Type of Surgery}

Eligible patients underwent CABG 175 (71.4\%), valvular repair $48(19.6 \%)$, or combination of both procedures $22 \quad(9 \%)$. The frequencies and corresponding percentages of each type of surgery are summarized in Fig. 3.

\subsubsection{Duration of Surgery}

The duration of the cardiac surgeries ranged from a minimum of 2 hours and a maximum of 10 hours. Majority of the surgeries had a duration ranging between 3 and 9 hours 177 (72.2\%), others ranged between 6 and 9 hours 55 (22.4\%), more than 9 hours

Table 1 Descriptive statistics of patients' age.

\begin{tabular}{lllll}
\hline Distribution & Minimum & Maximum & Mean & Standard deviation \\
\hline Age (Years) & 27 & 83 & 59.51 & 12.15 \\
\hline
\end{tabular}

Table 2 Patients characteristics.

\begin{tabular}{lll}
\hline Characteristic & & \\
\hline Age & Mean years $( \pm$ SD) & 59.51 years $( \pm 12.15)$ \\
Sex- no. (\%) & Male & $152(62)$ \\
& Female & $93(38)$ \\
Body Mass Index (BMI)- no. (\%) & Normal weight & $47(19.2)$ \\
& Overweight & $84(34.4)$ \\
& Obese & $72(29.4)$ \\
Allergy - no. (\%) & Contrast dye & $8(3.3)$ \\
& B-Lactam allergy & $6(2.4)$ \\
& Aspirin & $1(0.4)$ \\
& Sulfa group & $1(0.4)$ \\
& Carbamazepine & $1(0.4)$ \\
Co-morbid diseases- no. (\%) & CAD (coronary artery disease) & $177(72.24)$ \\
& Valvular heart disease & $41(16.73)$ \\
& CAD and valvular heart disease & $27(11.02)$ \\
& Hypertension & $159(64.9)$ \\
& Diabetes mellitus type 2 & $89(36.3)$ \\
& Dyslipidemia & $55(22.4)$ \\
\hline
\end{tabular}




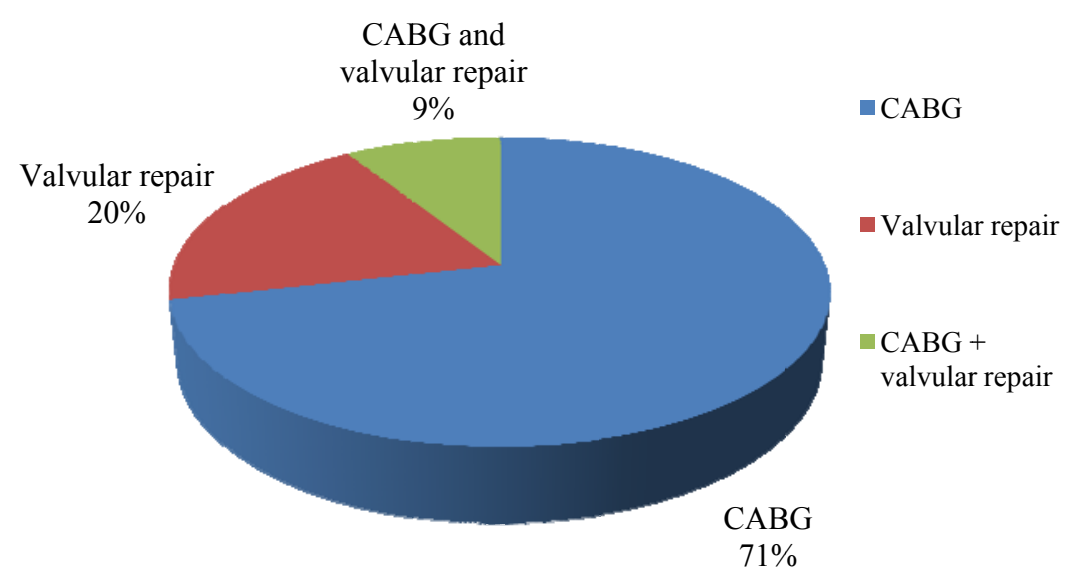

Fig. 3 Type of cardiac surgery.

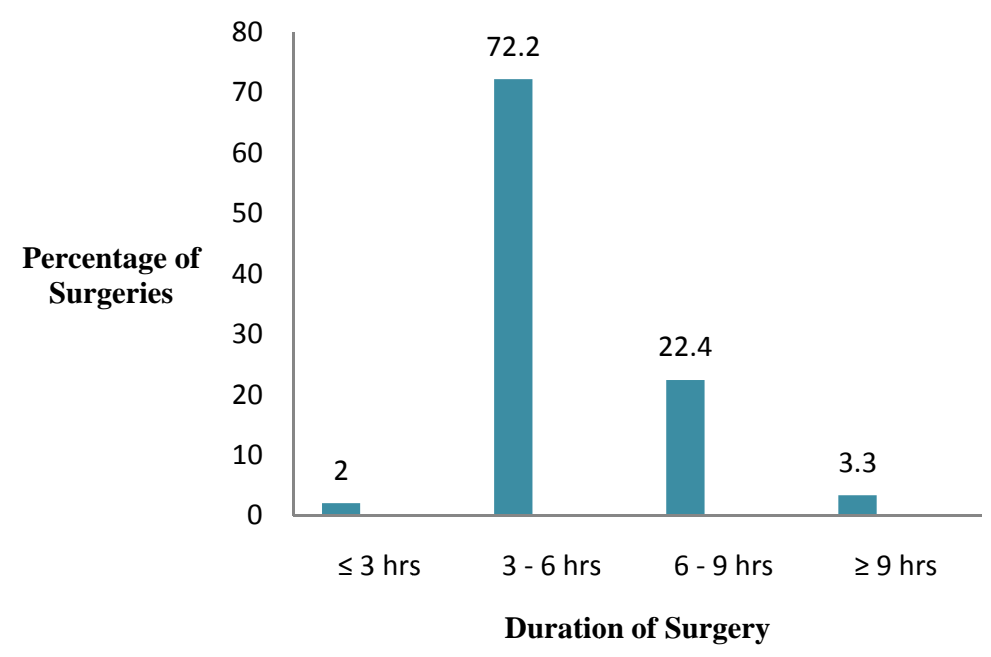

Fig. 4 Duration of cardiac surgery.

Table 3 Antibiotic regimens.

\begin{tabular}{lll}
\hline Antibiotic regimens & Frequency & Percent \\
\hline Vancomycin and ceftriaxone & 188 & 76.7 \\
Vancomycin and cefuroxime & 15 & 6.1 \\
Ceftriaxone & 13 & 5.3 \\
Vancomycin & 12 & 4.9 \\
Cefuroxime & 6 & 2.4 \\
Clindamycin & 2 & 0.8 \\
Others & 9 & 3.7 \\
Total & 245 & 100 \\
\hline
\end{tabular}

Table 4 Prophylaxis duration.

\begin{tabular}{lllll}
\hline Descriptive Statistics & Minimum & Maximum & Mean & Standard deviation \\
\hline Duration (Days) & 1 & 7 & 2.85 & 2.08 \\
\hline
\end{tabular}


$8(3.3 \%)$, and less than or equal to 3 hours $5(2 \%)$ (Fig. 4).

\subsection{Surgical Infection Prophylaxis Protocols}

\subsubsection{Route of Administration}

As recommended by the guidelines, all patients were given the antibiotic prophylactic regimens through the appropriate intravenous route.

\subsubsection{Choice of Antibiotic}

Enrolled participants were on different antibiotic regimens as shown in Table 3, where $188(76.7 \%)$ patients were on vancomycin and ceftriaxone, 15 (6.1\%) on vancomycin and cefuroxime, $13(5.3 \%)$ on ceftriaxone, $12(4.9 \%)$ on vancomycin, $6(2.4 \%)$ on cefuroxime, $2(0.8 \%)$ on clindamycin, and the others were on combination of gentamicin or teicoplanin and a cephalosporin.

Only twelve (4.9\%) from the enrolled patients were consistent with the treatment guidelines regarding the choice of the antibiotic. Six of those patients were on cefuroxime, and the others had PNC allergy so vancomycin or clindamycin was prescribed.

\subsubsection{Dosing and Redosing of Antibiotics}

Dosing appropriateness was studied for the patients who were given the recommended antibiotic regimen (12 participants). Proper dose was given in only seven patients $(58.7 \%)$. Five patients $(2 \%)$ out of 245 required redosing and four were correctly redosed.

\subsubsection{Preoperative Timing}

Prophylactic regimens consistent with the guidelines were given vancomycin within 120 minutes prior to surgery and 60 minutes for the others. However, when vancomycin was combined with other antibiotics, both drugs were administered within 120 minutes of incision which was not appropriate, and took place in 202 cases $(82.4 \%)$.

\subsubsection{Duration of Prophylaxis}

Prophylaxis was extended beyond one day in 173 patients $(70.61 \%)$ and the average duration was 2.85 days \pm 2.08 (Table 4 ).

\section{Discussion}

\subsection{Overview}

Antimicrobial prophylaxis is selected based on the most likely infecting organism where gram-positive bacteria as Staphylococcus aureus and Staphylococcus epidermidis are the main isolated pathogens [12]. Although cardiac surgery is generally considered a "clean" surgery with an intrinsically low infection rate (less than 5\%), postoperative infections complicating cardiac surgery are associated with potentially serious adverse outcomes [13,14]. Despite the large number of prospective, randomized, controlled clinical studies using various antimicrobials alone or in combination (including penicillins; first-, second- and third-generation cephalosporins; aminoglycosides; and vancomycin), no single regimen has emerged as clearly superior in preventing postoperative infections [14]. First- or second-generation cephalosporins (cefazolin, cefuroxime) are among the most effective antimicrobials in reducing postoperative infections in cardiac surgery and are the agents of choice according to the 2013 ASHP (American Society of Health-System Pharmacists). Data supporting the benefit of extending the antibacterial spectrum with combination antimicrobial prophylaxis are limited [15]. A 2002 cardiac surgery study conducted in Canada demonstrated that cefazolin was the antimicrobial used in $88 \%$ (38 of 43 ) of the cases, antimicrobial prophylaxis was initiated in the operating room $72 \%$ (26 of 36) of the time, and intraoperative supplemental antimicrobial doses were administered for cardiac procedures that took longer than a median of $4 \mathrm{~h}$ (range from 4 to $8 \mathrm{~h}$ ) [16]. Overall, the median duration of antimicrobial prophylaxis treatment was $36 \mathrm{~h}$ (range from 8 to 96 h). However, the results of a 2010 study in Australia that compared consistency with the guidelines between the years 2004 and 2008, reported protocol use increased from $58 \%$ to $80 \%(P=0.02)$, while concordance with version 13 of the Australian 
Therapeutic guidelines: antibiotic for both choice of agent and timing (duration of administration) remained around $10 \%$. Use of multiple agents was common, as was continued antibiotic administration after completion of surgery. Over 4 years, the proportion of cardiac surgical units reporting vancomycin administration for routine valve surgery prophylaxis doubled to $62 \%(P<0.001)[17]$.

This study was consistent with studies done abroad and demonstrated that there is excessive use of broad spectrum antibiotics and multiple agents, in addition to prolongation of the prophylaxis duration.

The study population consisted of males (62\%) with an average age of $59.51 \pm 12.15$ undergoing $\mathrm{CABG}$ (71\%).

The appropriate choice of antibiotic was given in only (4.9\%) of the cases and multiple agents were used in $(86.4 \%)$ of the surgeries. Vancomycin was commonly administered, and the duration of prophylaxis was extended beyond 1 day with an average duration of 2.85 days \pm 2.08 .

\subsection{Limitations}

A major limitation in our study was lack of surveillance data to asses if there was a real need for the use of such wide spectrum antibiotics. Also, the study was retrospective that relied on accuracy of written records where important data may have not been available. It is difficult to control bias and confounders, establish cause-effect relationships, and results are basically hypothesis-generating. Lastly, our outcomes lack external validity given the fact that it was a single center study.

\subsection{Strengths}

Our study is the first of its type in Lebanon where the main focus was on surgical prophylaxis in cardiac surgeries. A previous study was performed and studied prophylaxis in all types of surgery and showed that antimicrobial prophylaxis in Lebanese hospitals was fairly adequate in terms of respecting indications for selected surgical procedures, and that improvement could be made by reducing the duration of prophylaxis and avoiding the use of broad-spectrum antibiotics [11].

\subsection{Implications for Clinical Practice}

More education and communication are required to improve antimicrobial prophylaxis in Lebanon to reduce risks of surgical site infections, prevent resistance, and limit costs potentially associated with antibiotic misuse. The role of clinical pharmacist may facilitate this process across all surgical disciplines through interventions that should be implemented to optimize the perioperative antibiotic prophylaxis in procedures.

\section{Conclusions}

In summary, this survey of cardiac surgical antibiotic use demonstrated a marked deviation from existing national guidelines.

In cardiac surgery, the choice of agent is often inappropriately broad where an increasing number of antibiotic agents, including vancomycin, were being administered as routine prophylaxis, and these tended to be continued beyond 24 hours in $70 \%$ of the cases. Thus, this study showed that in cardiac surgeries, the optimal choice of antibiotics is seldom administered, duration of prophylaxis is excessively long, and the preoperative dose timing is rarely employed.

In conclusion, the results provide evidence that health care providers should be aware of their larger role in reducing unnecessary and inappropriate prescription of antibiotics prophylaxis in patients prior to surgery. There is a clear and urgent need for additional efforts and educative interventions to improve antibiotic prophylaxis to contribute to the prevention and control of SSIs. It is important to improve conformity with optimal surgical antibiotic prophylaxis policies so as to reduce risk of SSI and to prevent resistance and costs potentially associated with antibiotic misuse. A focus on the cause of errors 
in the system rather than mistakes of individuals is needed. A follow-up survey should be planned in one year to determine whether this information improved practice patterns.

\section{References}

[1] Ronald, L. N. 2001. "Preventing Surgical Site Infections: A Surgeon's Perspective.” Emerging Infectious Diseases 7: 2 .

[2] Doharty, G. M., and Way, L.W. 2006. Current Surgical Diagnosis. (12th ed). USA: McGraw Hill, 106-7.

[3] Mangram, A. J., Horan, T. C., Pearson, M. L., Silver, L. C., Jarvis, W. R., and the Hospital Infection Control Practices Advisory Committee. 1999. "Guideline for Prevention of Surgical Site Infection." Infect Control Hosp Epidemiol 20: 250-78.

[4] Harrington, G., Russo, P., Spelman, D., Borrell, S., Watson, K., Barr, W., Martin, R., Edmonds, D., Cocks, J., Greenbough, J., Lowe, J., Randle, L., Castell, J., Browne, E., Bellis, K., and Aberline, M. 2004. "Surgical Site Infection Rates and Risk Factor Analysis in Coronary Artery Bypass Graft Surgery." Infect Control Hosp Epidemiol 25: 472-6.

[5] Fowler, V. G. Jr., O’Brien, S. M., Muhlbaier, L. H., Corey, G. R., Ferguson, T. B., and Peterson, E. D. 2005. "Clinical Predictors of Major Infections after Cardiac Surgery.” Circulation 112 (9 Suppl): I35865.

[6] Eklund, A. M., Lyytikäinen, O., Klemets, P., Huotari, K., Anttila, V. J., Werkkala, K. A., and Valtonen, M. 2006. "Mediastinitis after More than 10,000 Cardiac Surgical Procedures." Ann. Thorac. Surg. 82: 1784-9.

[7] Anubhav, G., Milind, P. H., Minati, C., Arti, K. and Akshay, K. B. 2010. "Comparison of $48 \mathrm{~h}$ and $72 \mathrm{~h}$ of Prophylactic Antibiotic Therapy in Adult Cardiac Surgery: A Randomized Double Blind Controlled Trial." J. Antimicrob Chemother 65: 1036-41.

[8] Bratzler, D. W., Dellinger, E. P., Olsen, K. M., Perl, T. M., Auwaerter, P. G., Bolon, M. K., Fish, D. N., Napolitano, L. M., Sawyer, R. G., Slain, D., Steinberg, J. P., and
Weinstein, R. A. 2013. "Clinical Practice Guidelines for Antimicrobial Prophylaxis in Surgery.” Am. J. Health-Syst. Pharm. 70: 195-283.

[9] Anderson, D. J., Kaye, K. S., David, C., Kathleen, M., Arias, M. S., Kelly, P., Helen, B., David, P. C., Susan, E. C., Erik, R. D., Victoria, F., Dale, N. G., Frances, A. G., Peter, G., Michael, K., Evelyn, L., Jonas, M., Leonard, A. M., Lindsay, N., David, A. P., Trish, M. P., Sanjay, S., Cassandra, D. S., Robert A. W., Robert, W., and Deborah, S. Y. 2008. "Strategies to Prevent Surgical Site Infections in Acute Care Hospitals." Infect Control Hosp Epidemiol 29 (suppl1): S51-61.

[10] Bratzler, D. W., and Houck, P. M. 2004. "Antimicrobial Prophylaxis for Surgery: An Advisory Statement from the National Surgical Infection Prevention Project." Clin. Infect. Dis. 38: 1706-15.

[11] Azzam, R., Hamadé, N., Bedrossian, N., and Assaad, C. 2002. "Survey of Antimicrobial Prophylaxis for Surgical Procedures in Lebanese Hospitals." J. Med. Liban. 50 (5-6): 211-5.

[12] Ariano, R., and Zhanel, G. 1991. "Antimicrobial Prophylaxis in Coronary Bypass Surgery: A Critical Appraisal.” Ann. Phamacother. 25: 478-84.

[13] Milano, C., Kesler, K., Nancy, A., Daniel, J. S., and Robert, H. J. 1995. "Mediastinitis after Coronary Artery Bypass Graft Surgery: Risk Factors and Long-Term Survival." Circulation 92: 2245-51.

[14] Nelson, R., and Dries, D. 1986. "The Economic Implications of Infection in Cardiac Surgery." Ann. Thorac. Surg. 42: 240-6.

[15] Kreter, B., and Woods, M. 1992. “Antibiotic Prophylaxis for Cardiothoracic Operations; Meta-Analysis of Thirty Years of Clinical Trials." J. Thorac. Cardiovasc. Surg. 104: 590-9.

[16] Paradiso-Hardy, F. L., Patti, C., Chantal, P., and Stephen, E. F. 2002. "A National Survey of Antimicrobial Prophylaxis in Adult Cardiac Surgery Across Canada." Canada Can. J. Infect. Dis. 13 (1) 21-7.

[17] Timothy, P. H., Jeffrey, J. P. et al: Robertson Antibiotic prophylaxis for cardiac surgery in Australia. MJA. 2010 February Volume 192 Number 3. 


\section{Appendix I}

Lebanese International University

School of Pharmacy

Assessment of surgical infection prophylaxis in cardiovascular procedures in Lebanese hospitals

Data Collection Sheet

Patient Demographics

Age:

Gender: $\square$ Male $\square$ Female

Weight:

Height:

BMI:

Classification:

Allergy (s): $\square$ No $\square$ Yes, Specify:

Alcohol use: $\square$ No $\square$ Yes $\square$ Ex-alcoholic

Tobacco use: $\square$ No $\square$ Yes $\square$ Ex-smoker

Current Medical Conditions

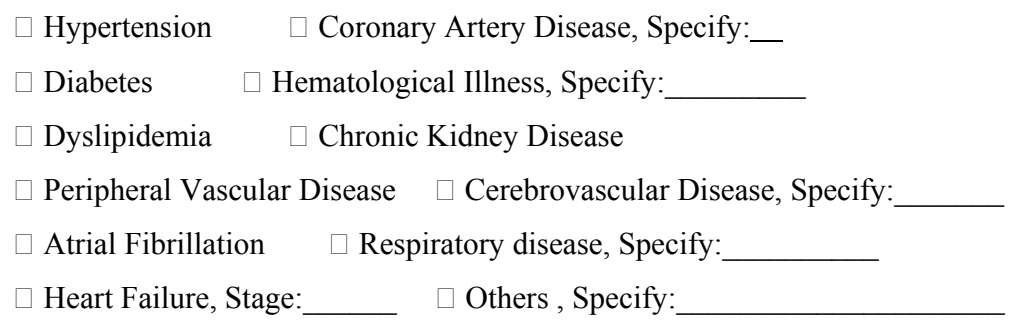

Type of Cardiac Procedure

$\square$ CABG $\square$ Cardiopulmonary bypass

$\square$ Valve replacement, Specify: $\square$ Cardiac device implants, Specify:

Duration of the Procedure

$\begin{array}{ll}\square 0-3 \mathrm{~h} & \square 3-6 \mathrm{~h} \\ \square 6-9 \mathrm{~h} & \square>9 \mathrm{~h}\end{array}$

Parenteral Prophylaxis

Preoperative

Medication:

Dose:

Start date:

Preoperative -dose timing:

Was the selection of the antibiotic appropriate? $\square$ Yes $\square$ No

If yes, answer the following:

Was the preoperative-dose timing appropriate? $\square$ Yes $\square$ No

Was the dosing of the antibiotic appropriate? $\square$ Yes $\square$ No

Was the duration of prophylaxis appropriate?

$\square$ Yes, $\square$ Single dose $\square$ Within $24 \mathrm{hr}$

$\square$ No, Duration :

Redosing:

Does the duration of surgery require redosing of the appropriate antibiotic? $\square$ Yes $\square$ No

If yes, was a redose given?

$\square$ Yes Medication:

Dose:

$\square$ No 